\title{
REGULIERUNGSTECHNIKEN FÜR UMWELTDELIKTE UND RESTORATIVE JUSTICE
}

\author{
REGULATORY TECHNIQUES FOR ENVIRONMENTAL CRIMES AND RESTORATIVE JUSTICE
}

Rafael Diniz Pucci*

\begin{abstract}
Zusammenfassung:
Die Normsetzung der transnational organisierten Umweltschäden, die mit der globalisierungsbedingten Komprimierung von Raum und Zeit angestiegen sind, wurde von verschiedenen theoretischen Perspektiven problematisiert. Auf der einen Seite wurden die auf der Common Law basierten Rechtssysteme grundsätzlich in Zusammenhang mit Regulierungstheorien verbunden, wie Restorative Justice, Smart Regulation, Command and Control und Compliance-Mechanismen. Auf der anderen Seite gibt es Theorien im Zusammenhang mit der Kriminalisierung solchen Verhaltens (wie z. B. die Grüne Kriminologie - Green Criminology).
\end{abstract}

Stichworte: Kriminologie. Umwelt. Umweltdelikte. Regulierung. Recht.

\begin{abstract}
:
The regulatory process of transnational organized environmental crimes, which have increased with the globalization-induced compression of time and space, has been object of different theoretical perspectives. By one side, the common law systems are in general designed through regulatory techniques such as Restorative Justice, Smart Regulation, Command and Control and Compliance. On the other hand, there are approaches related to the criminalization of the environmental deviance (such as Green Criminology Theories).
\end{abstract}

Keywords: Crime. Environment. Restorative Justice. Smart Regulation. Command and Control.

1. Kriminologische Umwelttheorien (Grüne Kriminologie/Green Criminology), kritische Theorie der Kriminologie, Konstruktivismus

Die Kriminologie und die Umwelt

Auf dem Gebiet der Kriminologie und der Strafrechtssoziologie gibt es ein erhebliches Defizit bei der Untersuchung der transnational organisierten Umweltschäden, trotz der intensiven sozialen Debatte über den Umweltschutz. Diese Debatte hat mit

Graduado pela Faculdade de Direito da Universidade de São Paulo (2005). Doutor em Filosofia e Teoria Geral do Direito pela Faculdade de Direito da Universidade de São Paulo (2008/2012). Professor Doutor Substituto de Economia Política Internacional no Departamento de Relações Internacionais da UNESP/ Franca ( $1^{\circ}$ semestre 2013). (dinizpucci@gmail.com). 
der Konferenz der Vereinten Nationen im Jahr 1972 in Stockholm begonnen und einen bedeutenden Fortschritt in der Konferenz im Jahr 1992 in Rio de Janeiro gebracht hat und verspricht neue Merkmale mit der Konferenz Rio + 20 im Jahr 2012. Darüber hinaus hat diese Diskussion tiefe Auswirkungen in politischen, gesetzlichen, sozialen, und wirtschaftlichen Bereiche hervorgerufen.

Die Kriminologie untersucht die abweichende Handlung und deren Protagonisten: Täter, Opfer und die Institutionen der sozialen Kontrolle. Außerdem handelt es sich um eine interdisziplinäre Wissenschaft, deren Ergebnissen von der Kriminalpolitik, dem Strafrecht und der Strafrechtsoziologie verwendet werden. ${ }^{1}$

In Unterschied zur Kriminalpolitik, die für das Gesetzgebungsverfahren Direktiven festlegt, begrenzt sich die Kriminologie auf die Beschreibung von Tatsachen. Anders als das Strafrecht, das den Tatbestand darstellt und Strafmaßnahmen vorschreibt, begrenzt sich die Kriminologie auf die Einrichtung von wiederkehrenden Standards der sozialen Abweichung. Im Gegensatz zur Strafrechtssoziologie, die die Verwirklichung der Strafnormen untersucht, erforscht die Kriminologie den Rechtspluralismus und die Bedingungen der Wirksamkeit, insbesondere welche abweichenden Folgen solche Phänomene verursachen können. ${ }^{2}$

Die Kriminologie hat historisch gesehen ihre Forschung in zwei großen Gebieten unterteilt:

1) Die Untersuchung der „Straßendelikte“, anders gesagt, die abweichenden Verhältnisse, die normalerweiser in der Gesellschaft beobachtet werden können. Eine große Rolle spielt hierbei die Täterschaft von machtlosen Individuen, die von den Apparaten der sozialen Kontrolle einfach kriminalisiert worden sind.

2) Die Untersuchung der White-Collar Crimes, die von den Tätern, die eine privilegierte Position im wirtschaftlichen, politischen oder sozialen Bereich innehaben, verursacht werden.

\footnotetext{
Vlg. KAISER, Gunther. Kriminologie, Heidelberg, C.F. Müller, 1993, S. 15. Vlg. auch SOUTH, Nigel. The ecocidal tendencies of late modernity: transnational crime, social exclusion, victims and rights. In: WHITE, Rob (Org.). Global environmental harm. Criminological Perspectives. Portland: Willan Publishing, 2010. p. 228, laut wem "criminology can be defined in varying ways but at its simplest and in the terms most commonly accepted it is taken to be the study of crime, criminals and criminal justice, although there are many different approaches to the subject. In fact, criminology as a field has always been shaped by the influence of, and borrowings from many other academic disciplines".

2 Vgl. BARATTA, Alessandro. Criminologia critica e critica del diritto penale. Introduzione alla sociologia giuridico-penale. Bologna: Il Mulino, 1982. SACK, F., DEICHSEL, W. Strafrechtssoziologie. In: KAISER, Gunther et al. (Orgs.). Kleines kriminologisches wörterbuch. Heidelberg: C.F. Müller, 1993. p. 500-507; auch KAISER, Gunther. Strafrechtssoziologie. Dimension oder Partitur der Kriminologie? Monatsschrift für Kriminologie und Strafrechtsreform, v. 62, S. 50-62, 1979, e FRITZ, Sack; RENÉ, König. Kriminalsoziologie. Frankfurt: Akademische Verlagsgesellschaft, 1974. S. 372-394.
} 
Die Bemühungen, die umweltschädlichen Handlungen im Bereich der Umweltkriminologie zu klassifizieren, haben offensichtlich in dieser Dichotomie Probleme gezeigt.

Tatsächlich ist die Dichotomie übermäßig in der Rolle des Täters konzentriert, was Begrenzungen bringt, da die umweltabweichenden Verhältnisse, besonders bei internationalen Delikten mehrere Agenten erfordern, oder eher Netze von Agenten, die nicht unbedingt in der Theorie der White-Collar Crime vorgesehen sind.

1.1. Grüne Kriminologie, kritische Kriminologie, Konstruktivismus und der Begriff der Umweltdelikte

Der Ausgangspunkt der Umweltkriminologie geht auf die methodologische Perspektive der kritischen Kriminologie (Critical Criminology) zurück. ${ }^{3}$

Die methodologische Folge davon ist signifikant, da die kritische Kriminologie sich an der dialektisch-materialistischen Methode orientiert. Natürlich erhält die „Grüne Kriminologie“ (Green Criminology) normalerweise als Folge des angenommenen methodologischen Maßstabs dieselben Kritiken, die auch die Kritische Kriminologie erfährt. ${ }^{4}$

Außerdem der theoretische Ausgangspunkt der grünen Kriminologie, deren Konsolidierung in der 1990er Jahren begann, ${ }^{5}$ ist die Bestätigung, dass es unmöglich ist, die Umweltzerstörung von der kapitalistischen Produktionsweisen und ihren Standards $\mathrm{zu}$ trennen.

Die Behauptung der „Grünen Kriminologie“, dass alle Umweltschäden zu kriminalisieren sind, egal, ob die Handlung legal oder illegal ist, wird kontrovers diskutiert. Auf diese Weise kümmert sich die grüne Kriminologie um alle schädlichen Verhältnisse, als Entscheidungen über Güteherstellung, was immer Umweltverantwortung mit sich bringt.

Auf diese Weise gilt der Begriff des Umweltdelikts für die grüne Kriminologie als ein erweiterter Begriff, da auch nicht-strafrechtliche Handlungen als Delikt gesehen werden können. In diesem Schritt kommt die Theorie des Konstruktivismus von Spector \& Kitsuse. ${ }^{6}$ In der Tat sieht die „Grüne Kriminologie“ das Delikt als eine soziale Konstruktion. So ist die Norm nicht mehr als eine Abstraktion, die der Realität nicht

\footnotetext{
3 Vgl. TAYLOR, Ian; WALTON, Paul; YOUNG, Jock. Critical criminology. Londres: Routledge, 1975.

4 Vgl. HALSEY, Mark. Against green criminology. The British Journal of Criminology, 2004.

5 Vgl. LYNCH, Michael. The greening of criminology. A perspective on the 1990's. The Critical Criminologist, v. 2, n. 3, S. 1-4;11-12.

6 Vgl. SPECTOR, Malcolm; KITSUSE, John. Constructing social problems. New York: Aldine de Gruyter, 1987.
} 
hundert Prozent entspricht. Deshalb muss man um die Umwelt deutlicher zu schützen, über die gesetzliche Regelungen hinausgehen, und alle Tatsachen beachten. Da das Opfer der Umweltschäden in der Regel die Gesellschaft ist, ist die Viktimisierung verbreitet und die strafrechtliche Verfolgung sinkt. Allerdings sind die Umweltschäden vielfältig, was die Nachfrage der Wiedergutmachung schwieriger oder manchmal sogar unmöglich macht. In diesem Sinne, könnten die Umweltbewegungen (insb. von Moral Claimers) und die Arbeit der politischen Parteien eine größere Rolle bei der Rekonstruktion der „Figur“ des Opfers spielen. ${ }^{7}$

\subsection{Moral Claimers und Moral Panics}

Die Legitimation der Konstruktion der sozialen Probleme wird heutzutage insbesondere im Umweltbereich in zwei Gebiete unterteilt: Zunächst muss die „wissenschaftliche Behörde“ ihr Gutachten abgeben. Bei dem Gutachten muss die „Behörde“ äußern, dass es sich um ein „Umweltproblem“ handelt und in wissenschaftliche Parameter klassifizieren. Dafür ist es notwendig, dass eine „Zusammenarbeit“ zwischen den Moral Claimers und den Wissenschaftlern stattfindet, weil die wissenschaftlichen Gutachten von den Moral Claimers ,akzeptiert“ werden müssen. Diese „Brücke“ wird normalerweise von Nicht-Regierungs Organisationen ,gebaut“.

Im Laufe der 90er Jahre bekamen Moral Claimers wie z.B. World Resource Institute, National Audubon Society, World Wildlife Fund und National Wildlife Federation hohe Zuschüsse von Unternehmen wie Cargill, Chevron, Ford und Motorola. Solche Investitionen könnten das Interesse solcher Moral Claimers an der Umwelt verringern.

Somit schließt die grüne Kriminologie, dass die Forderung wurde in der 70er. Jahre erweitert, um Menschen green zu „denken und kaufen“ zu ermutigen.

Obwohl es in Rahmen der Kriminalisierung der Umweltdelikte Direktiven gibt, sowohl auf internationaler Ebene als auch innerhalb Europas, kann man kaum eine konkrete Definition des ,Eco-Crime-Begriffes “ finden. ${ }^{8}$

\footnotetext{
7 Vgl. SOUTH, Nigel. The ecocidal tendencies of late modernity: transnational crime, social exclusion, victims and rights. In: WHITE, Rob (ed.). Global environmental harm. Criminological Perspectives. Portland: Willan Publishing, 2010. S. 228; 242: "The response requires a new academic way of looking at the world but also a new global politics. This is not to equate a green criminology with green party politics but instead to seek the articulation of a position premised on the principles of environmentalism and broader issues of environmental justice. Such a position should provide a standpoint going beyond the narrow boundaries of traditional criminology and draw together political and practical action to shape public policy".

8 Vgl. WALTERS, Reece. Eco crime and genetically modified food. New York: Routledge, 2011. S. 15.
} 
Um auf die Theorie des Konstruktivismus zurückzukommen, ${ }^{9}$ so ist es interessant zu bemerken, dass die Öffentliche Meinung zu "a form of science (...) a mean of political persuasion, a method of risk measurement and management and a means of social resistance" wird. Die signifikante Veränderung der Wissenschaft, die zunehmend privatisiert und politisiert wurde, hat in diesem Sinn die Bedeutung der Öffentlicher Meinung und auch der Media vergrößert. In der Tat hat die Verminderung der Bedeutung der Wissenschaft als nach WALTERS Meinung nachfolge, dass die "common sense, caution and public opinion become important aspects for policy-making and regulation". ${ }^{10}$

\subsection{Die Umwelt Gerechtigkeit (Environmental Justice)}

Die gesetzliche Entscheidung, welche umweltschädigenden Handlungen kriminalisiert werden sollten, basiert auf der Beziehung zwischen Macht und Unterdrückung. ${ }^{11}$

Auf diese Weise gibt es Gebiete innerhalb der grünen Kriminologie, wie das ecofeminism, ecoracism und red-green, die sich in dem Begriff Environmental Justice sammeln, um die Untersuchung von solchen Situationen durchzuführen. Der Ausgangspunkt ist, dass die unterentwickelten Länder von umweltschädigenden Tätern gewählt wurden, weil es aus ökonomischer Sicht sinnvoller ist, in unterentwickelten Ländern zu produzieren, da die Kosten mit für Umweltschutz gering sind und weniger Umweltnormen existieren. Als Beispiel nennt das ecoracism die umweltschädigenden Handlungen in afrikanischen und lateinischen Gemeinschaften oder sogar in IndianerTerritorien. ${ }^{12}$

In diesem Sinn schätzt die grüne Kriminologie, dass die Entscheidung gentechnisch veränderte Lebensmittel in Afrika herzustellen überhaupt keinen Bezug mit dem Hunger in der Region hat. In der Tat dient der Hunger als Ausrede von multinationalen Unternehmen, um die Herstellung von GV in der Region zu legitimieren. Folglich sieht die grüne Kriminologie solcher Handlung als einem Delikt. Und nicht nur die Unternehmen

9 Vgl. Id. Ibid., S. 51.

10 Vgl. Id. Ibid., S. 51-52.

11 Vgl. WHITE, Rob, Crimes Against Nature. Environmental criminology and ecological justice. Portland: Willan Publishing, 2009. S. 105, verbis: "green criminology. When it was first coined by Michael lynch in 1990 it was a term designed to harness green environmentalism and green political theories to examine environmental destruction as an outcome of the structure of modern capitalist production and consumption patterns. In this sense, green criminology has its theoretical roots embedded within the traditions of radical criminological schools of thought such as feminism, Marxism and social constructionism that arose as an opposition to dominate positivist ideologies arguing, among other things, that crime is to be found in relations of power, oppression and selective processes of criminalization".

12 Vgl. LYNCH, Michael; STRETSKY, Paul. The meaning of green: Contrasting criminological perspectives. Theoretical Criminology, v. 7, 2003. Vgl. auch den Brundtland Bericht. 
sind schuldig, sondern auch Staaten, beispielweise Regierungen, wie die US-Regierung, die den Hunger in afrikanischen Ländern verwendet, um transgenetische Experimenten auszuüben. ${ }^{13}$

\subsection{Die Bereiche der Grünen Kriminologie}

Synthetisch wurde die grüne Kriminologie in drei Hauptbereiche unterteilt:

A) Die Untersuchung der Bedeutung der Umweltkriminalität. Wie bereits erklärt, liegt die Auseinandersetzung bei der Erweiterung des Begriffes des Umweltdeliktes hier. Die Frage ist aber: Sind nur die Handlungen, die in strafrechtlichen Normen als Delikte dienen, Delikte? Oder können auch andere Handlungen, die umweltschädigend sind, als Delikt genommen werden ${ }^{14}$

B) Zweitens ist es die Aufgabe, das passende Instrument zur sozialen Kontrolle für die Vermeidung von Umweltschäden zu finden. Dazu gehört die Beschreibung der Maßnahmen, die im Regulierungsbereich aufgenommen werden.

C) Drittens wurde untersucht, wie man umweltschädigende Handlungen strafbar machen kann. Dafür werden die Ursachen und Bedingungen untersucht, die für die Kriminalisierung von umweltschädigenden Handlungen nötig sind. Und hier spielen eine wichtige Rolle die Moral Claimers, nämlich unterschiedliche Protestgruppen, die sich für die Kriminalisierung, aber auch für die NichtKriminalisierung oder sogar für die Entkriminalisierung einsetzen.

Kurz befasst sich die grüne Kriminologie mit dem Begriff des Umweltdelikts, die Festlegung ihrer Art und auch die Art der umweltsozialen Kontrolle vor allem im Hinblick auf die „Environmental Justice“.

Zusammen mit der Methodik der kritischen Kriminologie befasst sich die grüne Kriminologie mit den Umweltstrukturen von Klasse, Ethnien und Geschlecht und den Mechanismen der umweltsozialen Kontrolle. Unter diesem Gesichtspunkt wird der Einfluss der wirtschaftlichen Organisationen bei der Umweltzerstörung untersucht. Darüber hinaus kümmert sich die grüne Kriminologie im Sinne des Rechtspluralismus um die Beziehung zwischen den transnationalen Konzernen und den Rechtsetzungsverfahren Lobby. ${ }^{15}$

13 Vgl. WALTERS, Reece. Eco crime and genetically modified food. New York: Routledge, 2011. S. 65.

14 Vgl. WHITE, Rob. Crimes against nature. Environmental criminology and ecological justice. Portland: Willan Publishing, 2009. S. 6, 27-28.

15 Vgl. LYNCH, Michael; STRETSKY, Paul. Green criminology in the United States. In: BEIRNE, Piers. South, Nigel, Issues in Green Criminology. Confronting harms against environments, humanity and other 
2. Regulierungstechniken

Innerhalb der „Civil-Law-Ländern“ gibt es einen zunehmenden Trend für die Benutzung von Regulierungstechniken und Arten von Selbstregulierung in bestimmten Sektoren der Gesellschaft. Das passiert auch im Umweltbereich. ${ }^{16}$ In diesem Sinn ist der Fall der Europäischen Union Standard setzend, nämlich weil vor kurzem eine publike Konsultation unter der Leitung der Europäischen Kommission eröffnet wurde. Die publike Konsultation geht um die Benutzung der Smart Regulation (,intelligente Regulierung") in mehreren Bereichen, wie der Umwelt.

Die Konsultation fand vom April 2010 bis zum Juni 2010 statt und hat als beschriebenes Ziel: „Mit der Konsultation möchte die Kommission Stellungnahmen für eine Mitteilung über intelligente Regulierung sammeln, die für Herbst diesen Jahres geplant ist. Wie von Präsident Barroso im Herbst 2009 in seinen politischen Leitlinien angekündigt, wird die Kommission in dieser Mitteilung die Prioritäten für intelligente Regulierung vorstellen.“

Wäre es aber vernünftig, die Umweltverantwortung auf dem Markt der Selbstregulierung zu überlassen? Das Thema wird unterschiedlich in den kapitalistischen Diskurs eingebunden.

Ein Beispiel ist die progressive Einbeziehung der sozialen und ökologischen Verantwortung, ${ }^{17}$ beispielsweise auf dem Finanzmarkt. Die Finanzinstitutionen haben eine Kreditpolitik eingeführt, die sich an sozialen, ökologischen und politischen Kriterien orientiert. Auch wurden Projekte im Zusammenhang mit der Umwelt gefördert.

Die regulatorischen Arten erscheinen in unterschiedlichen Aspekten: Responsive Regulation, Tit for Tat, Pyramide, Smart Regulation, Command and Control und auch Restorative Justice (dessen exponentieller Theoretiker John Braithwaite will jetzt auch im Umweltbereich die Technik anwenden). ${ }^{18}$

\subsection{Restorative Justice}

Der von Nils Christie 1977 geschriebenen Aufsatz Conflict as Property, der im The British Journal of Criminology veröffentlichet wurde, hat die Restorative Theorien ausgelöst. Die Arbeit schenkt folgende Erkenntnis: In der Regel werden soziale

animals, Portland: Willan Publishing, 2007. S. 248-249.

16 BAUMOL, William. On the increasing role of economic research in management of resources and protection of the environment. Annual Review of Resource Economics, v. 2, S. 1-11, 2010.

17 SHAMIR, Ronen. Capitalism, governance, and authority: the case of corporate social responsibility. Annual Review of Law and Social Sciences, v. 6, S. 53, 2010.

18 BRAITHWAITE, John. Setting standards for restorative justice. The British Journal of Criminology, v. 42 , S. 563-577, 2002. 
Konflikte nicht von den ursprünglichen Protagonisten (zum Beispiel Täter und Opfer) gelöst, sondern durch einen Dritten (zum Beispiel den Staat).

So wird die Notwendigkeit erkannt, zu den ursprünglichen Teilnehmern des Konflikts zurück zu kehren. Die Erziehung würde in diesem Fall eine große Rolle spielen. In diesem Sinn bezieht sich Nils Christie auf die pädagogischen Theorien von Paulo Freire und Ivan Illich. Die Ergebnisse solcher Theorien sollten bei der sozialen Kontrolle der Kriminalität berücksichtigt werden. ${ }^{19}$

Die Theorie geht davon aus, dass der Staat den Konflikt verdinglicht, so dass sich um den Konflikt als staatliches „Eigentum“ gekümmert wird. In diesem Sinn wird der Konflikt aus der Sphäre der ursprünglich Beteiligten (Täter und Opfer) entnommen. Die innovative Idee Nils Christies war damals, dass die ursprünglichen Teilnehmer des Konflikts das Recht bekommen, ihren Konflikt selber zu lösen.

Das Problem ist, dass die Theorie mit der Idee des individuellen Subjekts arbeitet. Insbesondere im Umweltbereich werden allerdings die modern abweichenden Handlungen nicht von individuellen Subjekten, sondern von kollektiven Tätern wie Unternehmen, staatlichen Organisationen oder anderen Vereinigungen durchgeführt. Die Theorie der Restorative Justice wurde hauptsächlich von Theoretikern wie John Braithwaite und Howard Zehr aus Australien und Neuseeland entwickelt.

Vor kurzem hat die renommierte Zeitschrift The British Journal of Criminology die fünf einflussreichsten Aufsätze der letzten fünf Jahrzehnte ausgewählt. Als den einflussreichsten Beitrag der 2000er Jahre wurde der vom John Braithwaite geschriebenen Aufsatz Setting Standards for Restorative Justice ausgewählt, neben dem von Nils Christie geschriebene Beitrag Conflict as Property, 1970er Jahre, dem von Richard Quinney, Is Criminal Behaviour Deviant Behaviour?, 1960er Jahre, dem von Jock Young, Radical Criminology in Britain, 1980er Jahre, und dem von David Garland, The Limits of the Sovereign State, 1990er Jahre. Das zeigt den bedeutenden Einfluss der Restorative Justice Theorie in den aktuellen wissenschaftlichen Debatten.

Nach John Braitwaite sollte die Restorative Justice, die zurzeit vor allem im Zusammenhang mit Jugendgewalt angewendet wird, auch in anderen Bereichen wie Umwelt und Finanzen ${ }^{20}$ benutzt werden.

\footnotetext{
19 CHRISTIE, Nils. Conflict as property. The British Journal of Criminology, v. 17, 1977, S. 1: "when Ivan Illich and Paulo Freire are listened to, and my impression is that they are, the crime control system will also become more easily influenced".

20 Vgl. BRAITHWAITE, John. Restorative justice for banks through negative licensing. The British Journal of Criminology, v. 49, S. 439-450, 2009. Der Theoretiker will die Technik sogar in dem Finanzmarkt anwenden.
} 
Das Schema basiert auf dem Begriff der „Responsive Regulation“, der einem Pyramidensystem entspricht. Im Falle der Nichteinhaltung (,, uncompliance “ oder „,noncompliance“) der Norm erfolgt die härtere Sanktion, die die strafrechtliche ist.

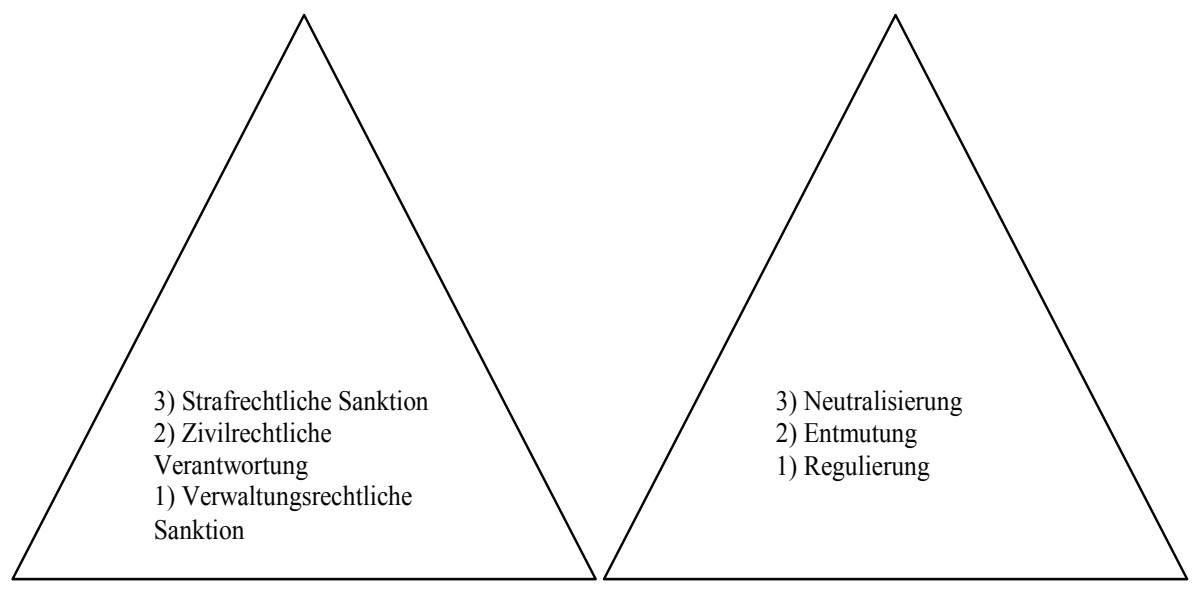

\subsection{Smart Regulation}

Die Smart Regulation beinhaltet nicht nur Arten der direkten Kontrolle im Sinn von „Command and Control“ sondern auch flexible Arten sozialer Kontrolle, die an Individuen, Organisationen oder Regierungen angewandt werden könnten. ${ }^{21}$

Es zeigt sich als Art von Selbstregulierung oder Co-Regulierung, die sich sowohl nach kommerziellen Interessen als auch nach den Interessen der Nicht-RegierungsOrganisationen richtet. Bei der Betrachtung von regulatorischen Defiziten, die in der Regel bei Umweltvorschriften zu beobachten sind, zeigt sich die Smart Regulation als effektive Art des Umweltschutzes.

Die Anhänger der Modelle verteidigen, dass die Strategie „third parties “ wie Unternehmen und NGOs ermutigen würde, die Umwelt zu schützen. Die Anhänger gehen davon aus, dass die Unternehmen damit ihre Kosten senken könnten und zusätzlich „Compliance“ mit bestehenden gesetzlichen Regelungen erhöhen würden (Win-WinSchema). Die publike Konsultation der Europäischen Kommission wies darauf hin, dass der private Sektor die intelligente Regulierung (Smart Regulation) stark unterstützt.

${ }^{21}$ GUNNINGHAM, Neil; SINCLAIR, Darren. Designing smart regulation. Oxford: Clarendon Press, 1998. 
Über dreißig Jahre ist die Umweltvorschrift durch den Einsatz von Techniken wie „command and control“ gekennzeichnet. Die Technik ist auf die Einhaltung von Mindeststandards festgelegt. In anderen Worten: Ohne mit Techniken, Technologien die minimalen „Commands“ zu erfüllen, würden die Unternehmen bestraft. ${ }^{22}$

Die Technik wurde für lange Zeit für ihre angebliche Wirksamkeit gelobt, besonders im Hinblick auf die Verringerung der Wasserverschmutzung und der Luft. Sie wurde aber wegen der Hemmung der Innovation, den hohen Kosten und der Inflexibilität vor allem durch Wirtschaftsfachmänner vielfach kritisiert. ${ }^{23}$

\subsubsection{Tit for tat}

Die Tit for Tat (TFT) dient als regulatorisches System, in dem die Aufsichtsbehörde und die Unternehmen zusammenarbeiten, so dass die Behörde konform mit den Unternehmen wird, wenn die Regeln vorher erfüllt wurden. ${ }^{24}$

\subsection{Regulierungstechniken und compliance}

Die Regulierungstechniken basiert auf der Idee der Einhaltung (,,Compliance“).Allerdings herrscht in der Regel im Umweltbereich die „Nichteinhaltung“ von Unternehmen im Hinblick auf Umweltstandards. ${ }^{25}$

Im Feld des Strafrechts sieht man tatsächlich immer mehr den Ersatz des Staates durch private Strategien der Selbst-Komposition bei Konflikten. Das geschieht besonders in bestimmten Bereichen wie Umwelt und White-Collar-Crimes, weil das Gesetzgebungsverfahren Lobbying erfordert.

So werden die Regulierungsstrategien dynamischer, die zivil- oder verwaltungsrechtliche Arten der Wiedergutmachung betonen.

São Paulo, januar de 2013.

22 ANGELO, Claudio. Guru de Chico Mendes critica lei florestal. Folha de S.Paulo, 16.06.2011.

23 GUNNINGHAM, Neil. Beyond compliance: next generation environmental regulation. Canberra: Australian Institute of Criminology, 2002.

24 SCHOLZ, John. Cooperation, deterrence, and the ecology of regulatory enforcement. Law and Society Review, v. 18, n. 2, 1984.

25 WOLF, Brian. Organized environmental crime. An analysis of Corporate Noncompliance with the Law, Lewiston/Queenston/Lampeter, The Edwin Mellen Press, 2009. 


\section{LITERATURVERZEICHNIS}

BARATTA, Alessandro. Criminologia critica e critica del diritto penale. Introduzione alla sociologia giuridico-penale. Bologna: Il Mulino, 1982.

BRAITHWAITE, John. Restorative justice for banks through negative licensing. The British Journal of Criminology, v. 49, S. 439-450, 2009.

CHRISTIE, Nils. Conflict as property. The British Journal of Criminology, v. 17, 1977.

HALSEY, Mark. Against green criminology. The British Journal of Criminology, 2004.

KAISER, Gunther. Strafrechtssoziologie. Dimension oder partitur der kriminologie? Monatsschrift für Kriminologie und Strafrechtsreform, v. 62, S. 50-62, 1979.

KAISER, Gunther. Kriminologie. Heidelberg: C.F. Müller, 1993.

LYNCH, Michael. The greening of criminology. A perspective on the 1990's. The Critical Criminologist, v. 2, n. 3.

SACK, Fritz; KÖNIG, René. Kriminalsoziologie. Frankfurt: Akademische Verlagsgesellschaft, 1974. S. 372-394.

SACK, F., DEICHSEL, W. Strafrechtssoziologie. In: KAISER, Gunther et al. (orgs.). Kleines Kriminologisches Wörterbuch. Heidelberg: C.F. Müller, 1993.

SOUTH, Nigel. The ecocidal tendencies of late modernity: transnational crime, social exclusion, victims and rights. In: WHITE, Rob (org.). Global environmental harm. Criminological perspectives. Portland: Willan Publishing, 2010.

SPECTOR, Malcolm; KITSUSE, John. Constructing social problems. New York: Aldine de Gruyter, 1987.

TAYLOR, Ian; WALTON, Paul; YOUNG, Jock. Critical criminology. Londres: Routledge, 1975.

WALTERS, Reece. Eco crime and genetically modified food. New York: Routledge, 2011. 